\title{
Detection of HCV-related Structures in the Mitochondria of Apoptotic Hepatocytes in Liver Biopsies from Chronically $\mathrm{HCV}$-infected Patients
}

\author{
Viviana Falcón*, Nelson Acosta-Rivero*, Glay Chinea*, Jorge Gavilondo*, María-C de la Rosa*, \\ Ivón Menéndez*, Santiago Dueñas-Carrera*, Ariel Viña*, Bienvenido Gra**, Miriam Noa***, Felix \\ Alvarez*, Josefina Rodríguez*, Juan Morales*
*Biomedicine Division, Center for Genetic Engineering and Biotechnology., P.O. Box 6162, C.P. 10600, Havana. Email: viviana.falcon@cigb.edu.cu
**Institute of Gastroenterology, C.P. 10400, Havana; C.P. 10400, Havana;
***National Center for Scientific Research, P.O. Box 6990, Havana.

The low levels of hepatitis $\mathrm{C}$ virus (HCV) particles in patient samples, the lack of a cell culture system supporting an efficient HCV replication or particle assembly, and the scarcity of EM positive reports on virus-like particles (VLPs) in liver biopsies from HCV infected patients, have hampered a better knowledge of the life cycle and pathogenicity of this virus [1]. However, detection and localization of HCV antigens in the liver would be important to study the host-viral interactions at the cellular level. Previously we have detected HCV VLPs inside hepatocytes cells from patients with chronic HCV infection [2]; in this work the presence of HCV-related structures in different compartments of hepatocytes was studied. Liver needle biopsies from eighth chronically HCVinfected patients were studied. In addition, samples were taken from two HCV-uninfected healthy donor livers as negative controls. Samples of liver biopsies were fixed in $0.2 \%$ glutaraldehyde and $4 \%$ paraformaldehyde in $0.1 \%$ OsO4 in the same buffer, dehydrated in ethanol, embedded in lowicryl. Ultrathin sections were incubated with anti-core (HCcAg) monoclonal antibodies (mAbs) recognizing the first 120 aa of HCcAg. The ultrathin sections were examined with a JEOL JEM 2000EX transmission electron microscope. In five of eight liver biopsies the presence of crystalloid bodies (CB) were observed in the cytoplasm of hepatocytes (Figure 1A). In some hepatocytes they also appeared in the mitochondria (M) (Figure 1B). These structures which are similar to the factories found in other viral infections [3], were not detected in hepatocytes from normal controls. To confirm the specificity of these observations, immunogold labelling with the various mAbs (arrows) was carry out in these samples and from normal controls. In all analyzed samples, $\mathrm{HCcAg}$ was specifically recognized in these crystal-associated particles (Figure 1C,D). However, $\mathrm{HCcAg}$ could not be detected by anti-HCcAg mAbs neither in samples from normal controls nor in sections incubated with normal mouse immunoglobulins as primary antibodies. In addition, virus-like particles (VLPs) with an average diameter of $50 \mathrm{~nm}$ were also observed in the mitochondria of some hepatocytes (Figures 1E). Interestingly, these VLPs were shown to interact with the nuclear chromatin (NC) in some apoptotic hepatocytes (Figure 1F). Of note, in these apoptotic cells either crystalloid bodies (CB) or VLPs were observed in the mitochondria. The presence of these HCcAgcontaining $\mathrm{CB}$ in the mitochondria of hepatocytes may be of relevance for $\mathrm{HCV}$ pathogenesis. Recently, it has been reported that HCcAg expressed in various cell lines localized in the mitochondria and causes mitochondrial injury, leading to oxidative stress [4]. Oxidative stress perturbs lipid metabolism, in addition to myriad other effects, thus contributing to steatosis and apoptosis, the latter being a standard feature of viral hepatitis. It is interesting to note, that in apoptotic hepatocytes (where either CB or VLPs were seen) VLPs interacting with nuclear chromatin were observed. Data suggest the possibility for a direct role of these HCV-related structures in apoptosis and pathogenicity. The crystal-associated particles found in this work are 
similar to those containing HCcAg-like particles obtained from recombinant $P$. pastoris cells [5]. They are also similar to the factories for nucleic acid replication and virion assembly found in other virus infections [3]. The location of these structures reflects the site of replication of a given virus. In addition the assembly of these new structures in the cell may alter or displace host-cell components and lead to one form of cytopathic effects. Thus, $\mathrm{HCcAg}$ protein could play an active role not only in $\mathrm{HCV}$ pathogenesis but also in HCV replication.

\section{References}

[1] C, Bosman, et al.. Res Virol (1998) 149, 311.

[2] V, Falcon, et al., Acta Microscopica (2001) Oct, 442

[3]. A. H, Sharpe, et al., Virology (1982); 120, 399.

[4] M, Okuda, et al., Gastroenterology (2002) 122, 366.

[5] V, Falcon, et al., Tissue and Cell (1999) 31, 117.

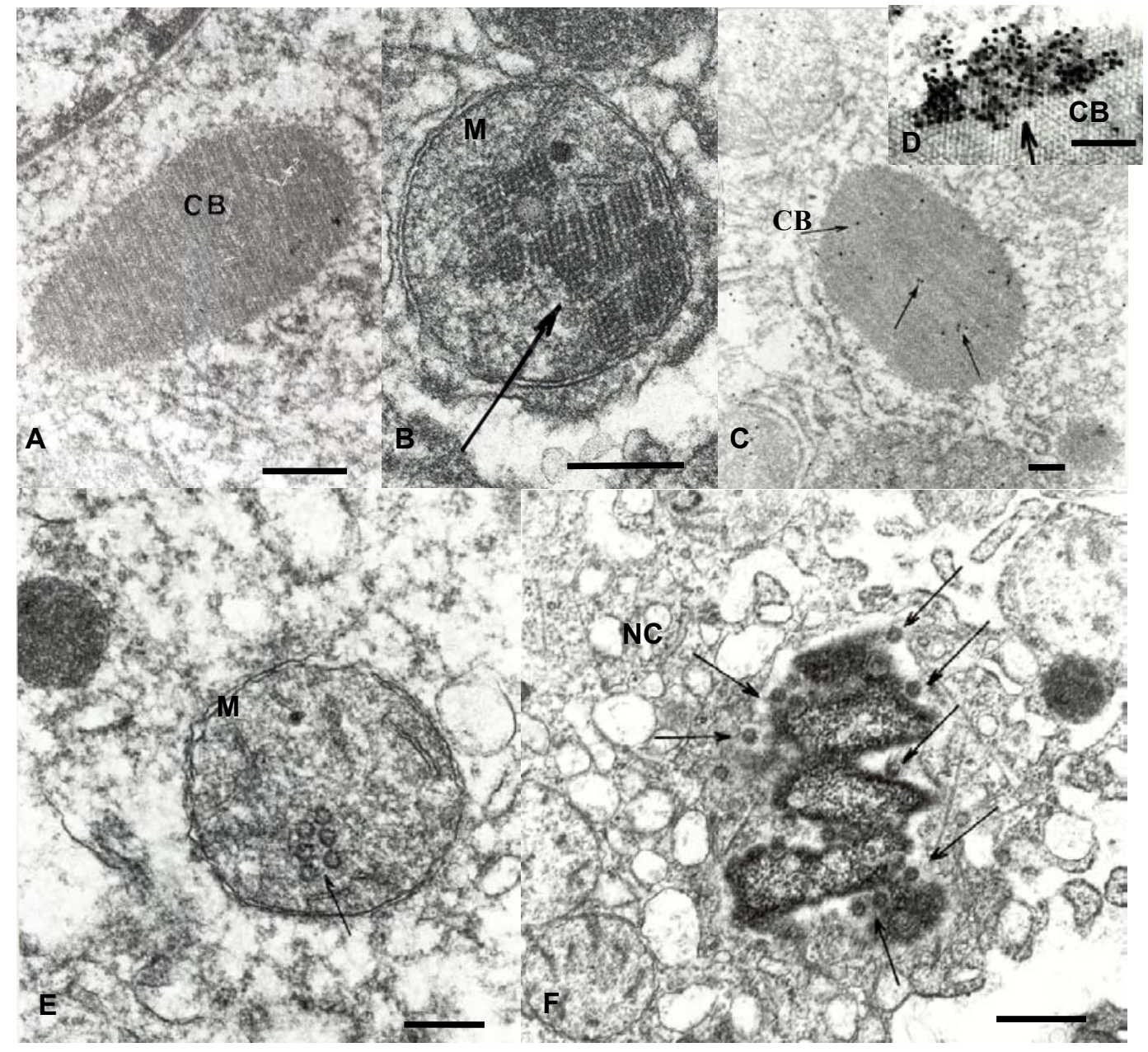

Figure 1. Bar $=500 \mathrm{~nm}$ in $\mathrm{A}$ and bars $=200 \mathrm{~nm}$ in $\mathrm{B}, \mathrm{C}, \mathrm{D}, \mathrm{E}, \mathrm{F}$. 